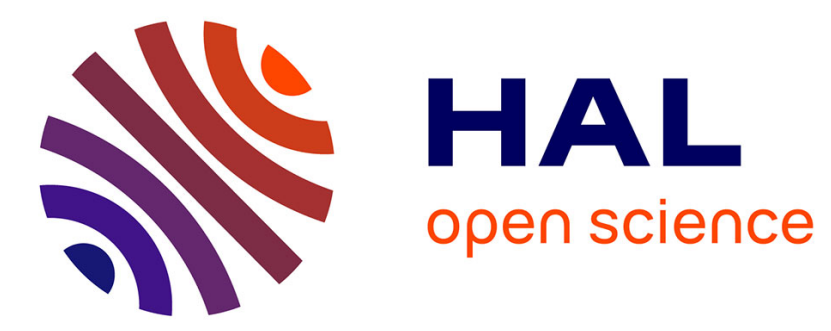

\title{
Statistics of macromolecular solutions trapped in small pores
}

\author{
M. Daoud, P.-G. de Gennes
}

\section{To cite this version:}

M. Daoud, P.-G. de Gennes. Statistics of macromolecular solutions trapped in small pores. Journal de Physique, 1977, 38 (1), pp.85-93. 10.1051/jphys:0197700380108500 . jpa-00208565

\section{HAL Id: jpa-00208565 https://hal.science/jpa-00208565}

Submitted on 1 Jan 1977

HAL is a multi-disciplinary open access archive for the deposit and dissemination of scientific research documents, whether they are published or not. The documents may come from teaching and research institutions in France or abroad, or from public or private research centers.
L'archive ouverte pluridisciplinaire HAL, est destinée au dépôt et à la diffusion de documents scientifiques de niveau recherche, publiés ou non, émanant des établissements d'enseignement et de recherche français ou étrangers, des laboratoires publics ou privés. 


\author{
Classification \\ Physics Abstracts \\ 7.146

\section{STATISTICS OF MACROMOLECULAR SOLUTIONS TRAPPED IN SMALL PORES}

\author{
M. DAOUD \\ Service de Physique du Solide et de Résonance Magnétique \\ Centre d'Etudes Nucléaires de Saclay, BP 2, 91190 Gif-sur-Yvette, France \\ and \\ P. G. DE GENNES \\ Collège de France \\ Place Marcellin Berthelot, 75231 Paris Cedex 05, France
}

(Reçu le 29 juin 1976, révisé le 25 août 1976, accepté le 2 septembre 1976)

\begin{abstract}
Résumé. - Nous analysons le comportement de solutions en bon solvant de chaînes macromoléculaires et confinées dans des tubes (ou des lamelles) de diamètre $D$ comparable au rayon de la pelote. Les interactions répulsives entre monomères sont prises en compte grâce à une méthode d'échelle qui va au-delà de la théorie usuelle de Flory-Huggíns.

Pour le problème des lamelles, nous trouvons cinq régimes différents (dépendant de la concentration $C$ et du diamètre $D$ ) avec des changements de comportement réguliers à toutes les frontières. Dans le problème des tubes un de ces régimes disparaît et deux lignes de cross-over se confondent, donnant lieu à une discontinuité plus marquée. Ceci est lié au fait que lorsque $D$ devient de l'ordre de la taille du monomère, les différentes pelotes ne peuvent plus s'interpénétrer.

Les propriétés thermodynamiques, les corrélations locales et la taille de la chaîne sont estimées dans chaque cas. Toutefois les arguments d'échelle prédisent seulement les lois de puissance (en $C$ et $D$ ) pour toutes ces quantités mais ne donnent pas les coefficients numériques.
\end{abstract}

\begin{abstract}
We analyse the theoretical behaviour of macromolecular chains dissolved in a good solvent, and confined into tubes (or slits) of diameter $D$ comparable to the coil radius. The repulsive interactions between monomers are taken into account by a scaling method which goes beyond the usual Flory Huggins approach.

For the slit problem, we find five different regimes (depending on the concentration $C$ and on the diameter $D$ ) with smooth cross-overs at all boundaries. For the tube problem, one of these regimes disappears and two cross-over lines merge, giving rise to a line of stronger discontinuity. This is related to the fact that, when $D$ decreases down to the monomer size, different coils cannot overlap each other at all.

For all regimes that thermodynamic properties, the local correlations and the overall chain size are estimated. The scaling arguments, however, predict only the power laws (in $C$ and $D$ ) for all these quantities, and do not give precise numerical coefficients.
\end{abstract}

1. Introduction. - The behaviour of flexible polymer chains moving inside porous structures is important for many practical problems = filtration, gel permeation chromatography, heterogeneous catalysis, oil recuperation, etc. A number of data have been taken both on partition coefficients - i.e. on the thermodynamic state of the trapped chains - and on diffusion properties [1]. The present paper presents some theoretical considerations on the first problem.

We consider only the case when the solution is contained in fine pores where the volume available to solvent and solute is limited by well defined boundaries. (Thus the problems of chains trapped in a swollen gel are not included.) We assume the simplest shapes for the pores : i.e. an infinite slit of width $D$ (Fig. 1a) or a long capillary of circular cross-section (Fig. 1b) $\left({ }^{1}\right)$. Case (a) might be found with a cleavage fracture in mica. Case (b) can be approximated by pores in leached glass [1] or with open crystallographic structures such as zeolites [2].

( $\left.{ }^{1}\right)$ The precise shape of the cross-section (circle, or square...) is unimportant for the scaling discussion to be described here. 

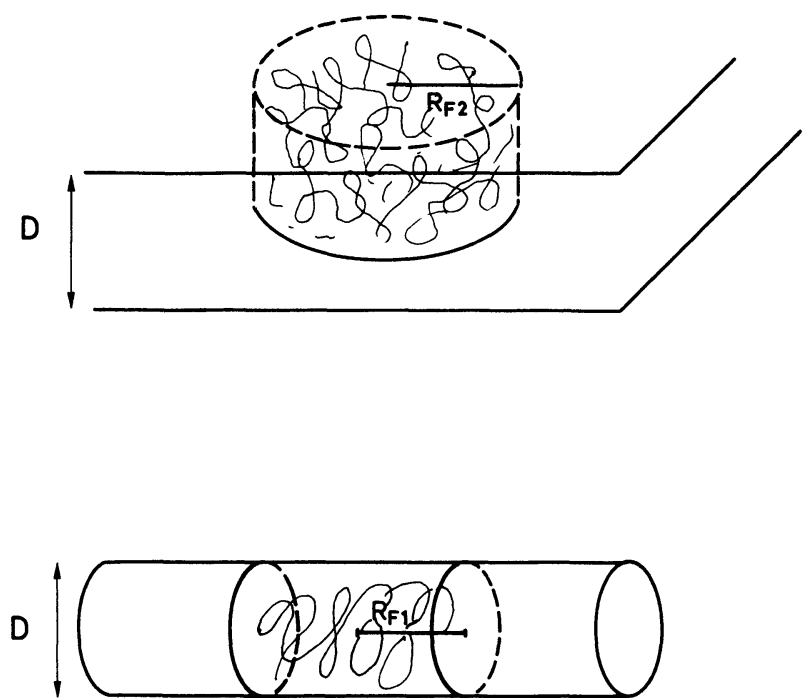

Fig. 1. - Conformations of a single flexible chain trapped into a slit (a) or a capillary (b).

We assume that polymer absorption on the solid surfaces is negligible $=$ this can be achieved on glass, for instance, through replacement of the $-\mathrm{OH}$ groups by trimethylsilane [3]. We also, restrict our attention to uncharged polymers. A good example for our purposes would be polystyrene dissolved in a good solvent for the bulk solutions of such systems we have detailed neutron data, which have shown qualitative deviations from the Flory Huggins theory [4]. These deviations have been interpreted by a scaling method.

Our aim is to extend these considerations to situations where the pore size $D$ is comparable to, or even smaller than the individual coil radius $R\left({ }^{2}\right)$. For a single ideal chain trapped in a pore the entropy and all related thermodynamic parameters have already been discussed in detail [5]. But the scaling approach described in ref. [4] allows us to do much more : we can incorporate rigorously the repulsive interactions between monomers, which are very important in practice, and treat both dilute solutions (where different chains do not overlap) and semi-dilute solutions (where the overlap is significant).

From a theoretical standpoint, these problems are interesting because they are associated with various cross-overs between three dimensional behaviour (for $R<D$ ) and two dimensional behaviour (for $R>D$ ) in a slit (or between dimensionalities 3 and 1 in a capillary).

There is a close relation between polymer statistics and magnetic phase transitions [6,7].

An abundant theoretical literature can be found on magnetic transitions in systems of restricted dimensionality $[8,9]$. However, only a small fraction of this work can be transposed immediately to the polymer problem. For this reason, we shall not emphasize very much the magnetic analogy, but we shall write

$\left.{ }^{2}\right)$ On the other hand, we usually assume that $D$ is much larger than the monomer size. down directly most of our scaling assumptions on the polymer system. This style (hopefully) should make our arguments more accessible to physical chemists. In section 2 we discuss the single chain problem (with excluded volume effects) for both slits and capillaries. In section 3 we consider overlapping chains in a slit; in section 4 we proceed to the most delicate case of overlapping chains in a capillary.

2. The single chain problem. -2.1 THE CHAIN IN OPEN SPACE. - Let us start from a single chain in an infinite sea of solvent (Fig. 2A). The situation is described by the following parameters :

- the ideal chain radius $R_{0}=N^{1 / 2} a$ where $N$ is the polymerization index and $a$ a characteristic length (which for brevity we call the monomer size),

- the excluded volume $v$ proportional to the parameter $1 / 2-\chi$ of Flory [10]. For the good solvents of interest here $v$ is positive and of order $a^{3}$. It is sometimes convenient to visualize this by imposing a lattice model, as in the Flory-Huggins theory [10] e.g. a cubic lattice of mesh size $a$; all chains are then represented by self avoiding random walks of $N$ steps on this lattice,

- the real radius of the chain $R_{\mathrm{F} 3}$ (where F stands for Flory and 3 for 3 dimensional) is then of the form [10]

$$
R_{\mathrm{F} 3} \cong a N^{3 / 5} .
$$

In eq. (2.1) (and in all what follows an $\cong$ sign) we purposely ignore all numerical coefficients.

2.2 Chain IN A SLIT. - Let us now bring the chain inside a slit of diameter $D$. If $D \gg R_{\mathrm{F} 3}$ no change in

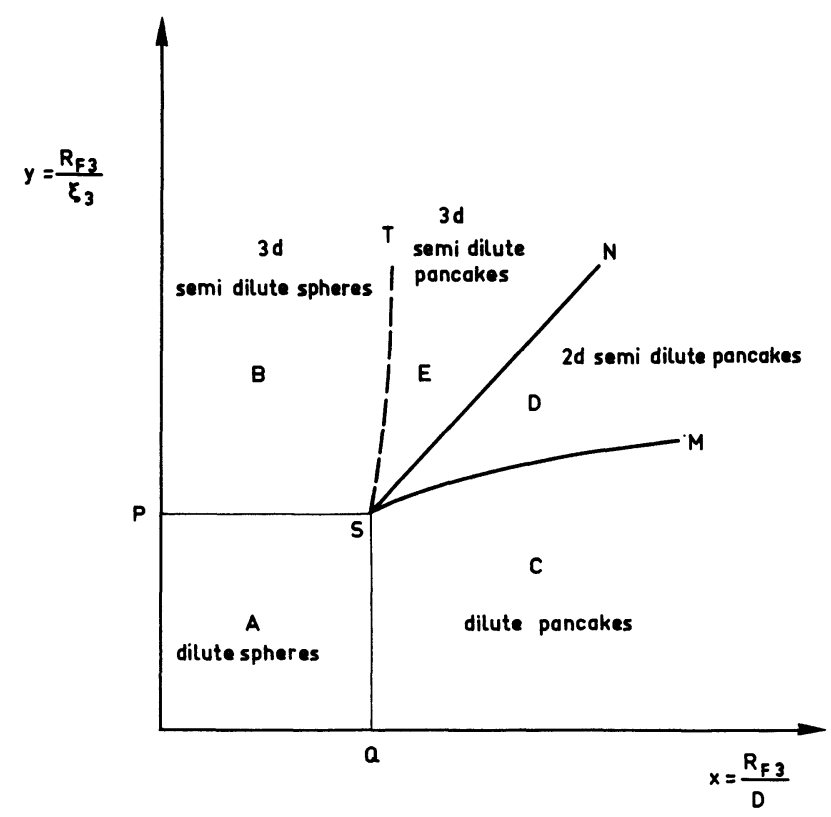

FIG. 2. - Various regimes for macromolecular solutions in a slit. $\xi_{3}$ is a characteristic length of bulk solutions, varying like the concentration $C^{-3 / 4}$. Thus the ordinates $y$ increase with concentration. The abscissas $x$ are inversely proportional to the slit thickness $D$. 
shape is expected and the chain remains a spherical coil. But if $D<R_{\mathrm{F} 3}$ a significant squeezing takes place; the coil becomes a flat pancake, of thickness $D$ and radius $R_{\mathrm{F} 2}$ (Fig. $1 a$ ). The Flory analysis tells us that for this two dimensional problem, $R_{\mathrm{F} 2}$ must be proportional to $N^{3 / 4}$. However, the coefficient here is non-trivial, since it will depend strongly on $D$. A scaling assumption on $R_{\mathrm{F} 2}$ can be formulated as follows

$$
R_{\mathrm{F} 2}=R_{\mathrm{F} 3} f\left(\frac{R_{\mathrm{F} 3}}{D}\right)=R_{\mathrm{F} 3} f(x)
$$

where the dimensionless function $f(x)$ has the following features

$$
\left.\begin{array}{l}
f(x \rightarrow 0)=1 \\
f(x \gg 1) \cong x^{m}
\end{array}\right\}
$$

$m$ being an exponent which is unknown at this stage. The second limit in (2.3) implies that for $D \ll R_{\mathrm{F}}$ :

$$
R_{\mathrm{F} 2} \sim R_{\mathrm{F} 3}^{m+1} D^{-m} \sim N^{\frac{3}{5}(m+1)} a^{m+1} D^{-m} .
$$

As explained above, the power of $N$ involved must be equal to $3 / 4$

$$
\frac{3}{5}(m+1)=\frac{3}{4} .
$$

Thus we must have $m=1 / 4$ and

$$
\frac{R_{\mathrm{F} 2}}{a} \cong N^{3 / 4}\left(\frac{a}{D}\right)^{1 / 4} \quad(\text { for } R>D) .
$$

This result can also be derived directly by an argument of the Flory type, where the free energy of the pancake is split into an elastic deformation energy and a repulsive term. We avoid this presentation however, because, although it gives the correct exponents it does not give the free energy itself correctly. This situation is now well recognized $[11,12]$.

Another approach to eq. (2.5) is the following : short segments of the chain (with a number of monomers $n$ smaller than a certain limit $n_{\mathrm{c}}$ ) will show internal correlations which are identical to those of a 3-dimensional self avoiding walk, and independent of the existence of a confining slit. But longer segments $\left(n>n_{\mathrm{c}}\right)$ will display certain two dimensional features. The cross-over point $n_{\mathrm{c}}$ is such that $n_{\mathrm{c}}^{3 / 5} a \cong D$ or $n_{\mathrm{c}} \cong(D / a)^{5 / 3}$.

For long range properties (distances $>D$ ) we can picture the chain as a sequence of $N / n_{\mathrm{c}}$ subunits, each of volume $D^{3}$, with complete mutual exclusion between subunits. The subunit centers are confined to the midplane of the slit, and each of them is associated with an impenetrable area $D^{2}$ in this plane. The resulting two dimensional self avoiding walk (with unit step $D$ ) has a size

$$
R_{\mathrm{F} 2} \cong D\left(\frac{N}{n_{\mathrm{c}}}\right)^{v_{2}}
$$

where $v_{2}$ is the two dimensional exponent, for which we use the (approximate) Flory value $v_{2}=3 / 4$. It can be checked that $\left(2.5^{\prime}\right)$ and $(2.5)$ coincide.

An interesting question is the form of the density across the slit. The density vanishes on both walls. For the present (single chain) problem we expect the density to reach its average value within a certain healing distance from the wall, which is a constant fraction of $D$ : i.e. there is no flat plateau in the profile. We shall see later that for the many chain problem, the situation is often different, and the healing length shorter.

Let us now quote the scaling form for the free energy per chain $\mathscr{F}_{N}$ measured from the state with $D=\infty$. It must be of the form

$$
\frac{\mathcal{F}_{N}}{k_{\mathrm{B}} T}=g\left(\frac{\boldsymbol{R}_{\mathrm{F3}}}{\boldsymbol{D}}\right)
$$

where $k_{\mathrm{B}}$ is Boltzmann's constant, and $g(x)$ is a dimensionless function.

$$
\left.\begin{array}{rl}
g(0) & \rightarrow 0 \\
g(x \gg 1) & \rightarrow x^{p}
\end{array}\right\} .
$$

The value of $p$ can be obtained from the requirement that $\mathcal{F}_{N}$ be an extensive function of $N$

$$
\begin{aligned}
\left(\frac{R_{\mathrm{F} 3}}{D}\right)^{p} & =N^{3 p / 5}(a / D)^{p} \\
\frac{3 p}{5} & =1 \\
\frac{\mathfrak{F}_{N}}{k_{\mathrm{B}} T} & \cong N\left(\frac{a}{D}\right)^{5 / 3}\left(R_{\mathrm{F} 3}>D\right) .
\end{aligned}
$$

Eq. (2.8) can also be derived, more fundamentally, from the magnetic analogy [8]. Note the difference with the result of ref. [5] for ideal chains : the latter reads

$$
\frac{\mathcal{F}_{N}}{k_{\mathrm{B}} T} \cong\left(\frac{R_{0}}{D}\right)^{2}=N\left(\frac{a}{D}\right)^{2} .
$$

In principle $\mathscr{F}_{N}$ is measured by comparison of concentrations in the pore and in a bulk solution, exchanging chains with the pore :

$$
\frac{C_{\text {pore }}}{C_{\text {bulk }}}=\alpha \exp \left(-\frac{\mathcal{F}_{N}}{k_{\mathrm{B}} T}\right) \quad \text { (dilute regime) } .
$$

The'prefactor $\alpha$ also depends on $x$, but the dominant factor is the exponential : thus $\mathscr{F}_{N}$ can be obtained, as described for instance in ref. [1]. The difference in exponents between eqs. (2.8) and (2.9) may however be too small to be seen.

2.3 Chain IN A Capillary. - The arguments are very similar and we shall quote only the results. For $R_{F 3}>D$ the chain is deformed into a long cigar 
of diameter $D$ and length $R_{\mathrm{F} 1}$ (Fig. $1 b$ ). The latter is linear in $N$

$$
R_{\mathrm{F} 1} \cong N a\left(\frac{a}{D}\right)^{2 / 3}
$$

The free energy change associated with the confinment is still described by eq. (2.8) and only the numerical coefficient (not quoted in (2.8)) will differ.

3. Overlapping chains in a slit. - 3.1 THE CRITICAL OVERLAP CONCENTRATION $C_{1}$. - We now have a reasonably good picture of the behaviour of a single chain. What happens if the concentration $C$ increases $\left({ }^{3}\right)$, so that different chains begin to overlap ? Clearly there will be a change of behaviour when different chains come into contact: this defines a critical overlap concentration

$$
C_{1}\left(\frac{R_{\mathbf{F}}}{D}\right)=C_{1}(x) .
$$

a) For $x<1, C_{1}(x)$ becomes identical to a concentration $C^{*}$ which has been discussed extensively in ref. [4]

$$
C^{*} \cong \frac{N}{R_{\mathrm{F} 3}^{3}} \cong a^{-3} N^{-4 / 5} .
$$

b) For $x>1 C_{1}(x)$ corresponds to a number $N$ of monomers in a volume of order $R_{\mathrm{F} 2}^{2} D$ where $R_{\mathrm{F} 2}$ is the single pancake radius calculated in eq. (2.5)

$$
\begin{aligned}
C_{1}(x) & \cong \frac{N}{R_{\mathrm{F} 2}^{2} D} \cong a^{-3} N^{-1 / 2}\left(\frac{a}{D}\right)^{1 / 2} \\
& \cong C^{*} x^{1 / 2} \quad(x \gg 1) .
\end{aligned}
$$

It is convenient to display results such as (2.2) on a diagram where one axis measures $x$, while the other axis measures the concentration or a related quantity. This diagram appears in figure 2 . Instead of the concentration, we use the variable

$$
y=\frac{R_{\mathrm{F} 3}}{\xi_{3}(C)} \cong\left(\frac{C}{C^{*}}\right)^{3 / 4}
$$

where $\xi_{3}(C)$ is the correlation length for bulk solutions of concentration $C$. The various meanings of $\xi_{3}(C)$ have been discussed in ref. [4], and will not be restated here in detail. The briefest summary is obtained through the concept of blobs [4], [12]. Each chain (in an overlapping, three dimensional solution) can be conceived as a sequence of blobs, each containing $g=C \xi_{3}^{3}$ monomers and each having a diameter $\xi_{3}$. Inside one blob excluded volume effects are important, but different blobs are essentially uncorrelated, and the chains are ideal at large scales. For future use it will

$\left(^{3}\right)$ We define $C$ as a number of monomers per unit volume. be convenient to rewrite the relation between $\xi_{3}$ and $C$ under the ( $N$ independent) form

$$
\xi_{3} \cong a\left(C a^{3}\right)^{-3 / 4} \text {. }
$$

Eqs. (3.1) and (3.2) for the critical overlap concentration are described by the line PSM on the diagram of figure 2. Below this line the solutions are dilute (non overlapping coils). Above this line they are semi-dilute.

We shall now consider the cross-over between dilute and semi-dilute behaviour. The first and most trivial case is obtained when $D$ is large - i.e. when we are dealing with normal 3 dimensional solutions. This corresponds to the line PS of figure 2. Below PS (region A) we are dealing with separate spherical coils of size $R_{\mathrm{F} 3}$. Above PS (region B) we have overlapping spherical coils, with a radius $R$ decreasing slowly with concentration, as explained in ref. [4]. In region B the description in terms of blobs is adequate.

In region A the osmotic pressure $\pi$ is comparable to the perfect gas pressure

$$
\frac{\pi}{k_{\mathrm{B}} T} \cong \frac{C}{N} .
$$

In region $\mathrm{B}$ the osmotic pressure shows a slight deviation from the Flory-Huggins exponent, and is given by

$$
\frac{\pi}{k_{\mathrm{B}} T} \cong \frac{1}{\xi_{3}^{3}} \cong \frac{1}{a^{3}}\left(C a^{3}\right)^{2.25} \quad(\text { region } \mathrm{B}) .
$$

Experimental checks on these various laws are presented in ref. [4].

3.2 SEMI-DILUTE SOLUTIONS WITH TWO DIMENSIONAL BEHAVIOUR. - We consider now region (D) in the diagram of figure 2 . Physically this means that starting with separate pancakes we increase the concentration slightly and force the pancakes to overlap. The crossover through the line SM can again be analised by scaling methods. The main features are the following.

a) There exists a correlation length $\xi_{2}$ in the slit plane, dependent on $C$ but independent of the polymerization index $N$. Just at the cross-over, $\xi_{2}$ must be equal to the radius of one isolated chain $R_{\mathrm{F} 2}$ (eq. (2.5)). These two requirements impose

$$
\xi_{2} \cong R_{\mathrm{F} 2}\left(\frac{C_{1}}{C}\right)^{3 / 2} \text {. }
$$

(The reader may check that with the forms (3.2) for $C_{1}$, and (2.5) for $R_{\mathrm{F} 2}$ the length $\xi_{2}$ is indeed independent of $N$.)

$b$ ) The overall length of one chain $R$ must vary like $N^{1 / 2}$ since each chain is ideal on spatial scales larger than $\xi_{2}$. $R$ must also reduce to $R_{\mathrm{F} 2}$ when $C=C_{1}$. This imposes

$$
\left.R \cong R_{\mathrm{F} 2}\left(\frac{C_{1}}{C}\right)^{1 / 2} \quad \text { (region } \mathrm{D}\right)
$$


c) The osmotic pressure $\pi(C)$ reflects the local correlations (on a scale $\xi_{2}$ or $D$ ) and is thus independent of $N$ in the semi dilute region. Furthermore $\pi$ must be comparable to the perfect gas pressure $k_{\mathrm{B}} T \frac{C}{N}$ on the dilute side. These two requirements impose

$$
\frac{\pi(c)}{k_{\mathrm{B}} T} \cong \frac{C_{1}}{N}\left(\frac{C}{C_{1}}\right)^{3} \cong \frac{1}{D \xi_{2}^{2}} \quad(\text { region } \mathrm{D}) .
$$

d) It must be emphasized that $\pi(C)$ is the osmotic pressure inside the slit : it is related to the change of free energy when the solution expands, always remaining inside a gap of fixed $D$. A more important thermodynamic parameter is the chemical potential $\mu$ (per monomer) which allows one to discuss the equilibrium between a slit (concentration $C$ ) and a bulk solution (concentration $C_{\mathrm{b}}$ ). In the latter system we may write

$$
\mu_{\mathrm{b}}=\mu_{\mathrm{o}}+k^{\prime} \frac{\pi_{3}\left(C_{\mathrm{b}}\right)}{C_{\mathrm{b}}}
$$

where $\mu_{0}$ is the potential of a single chain, $\pi_{3}$ is given by eq. (3.4b) and $k^{\prime}$ is a numerical constant. This simple scaling form will hold in the semi-dilute region. In region $\mathrm{D}$ we may write

$$
\mu_{\mathrm{D}}=\mu_{0}+k^{\prime \prime}\left(\frac{a}{D}\right)^{5 / 3} k_{\mathbf{B}} T+k^{\prime \prime \prime} \frac{\pi(C)}{C}
$$

where the second term represents the work (per monomer) for squeezing a coil into a pancake (eq. (2.8) divided by $N$ ) $k^{\prime \prime}$ and $k^{\prime \prime \prime}$ are numerical constants. The last term represents the work required to bring the different coils in close overlap. $\pi(C)$ is given by eq. (3.7).

As we shall see later, in region $\mathrm{D}$ we always have constantly $\xi_{2}>D$. When this inequality is inserted into eqs. (3.7), (3.9) it can be checked that the $k^{\prime \prime \prime}$ term in (3.9) is always smaller than the $k^{\prime \prime}$ term.

Setting $\mu_{\mathrm{b}}=\mu_{\mathrm{d}}$ we arrive at an equilibrium condition of the form

$$
\left.\left(\frac{C}{C_{1}}\right)^{2} \cong k_{1} N\left(C_{\mathrm{b}} a^{3}\right)^{1.25}-k_{2} x^{5 / 3} \quad \text { (region } \mathrm{D}\right)
$$

where $k_{1}$ and $k_{2}$ are two numerical constant. Eq. (3.10) could in principle be checked on suitable porous media, using the techniques described in ref. [1].

3.3 SeMi-Dilute SOlutions With 3-Dimensional LOCAL BEHAVIOUR. - It is important to realize that the behaviour associated with region (D) cannot extend up to very high concentrations. For instance, if we take eq. (3.6) for the chain size, and try to apply it to a molten polymer problem $\left(\mathrm{Ca}^{3}=1\right)$ we get $R^{2} \cong N a^{2}(a / D)^{1 / 2}$ while the correct result is

$$
R^{2} \cong N a^{2} \text {. }
$$

Thus a second cross-over must occur, at a concentration $C_{2}(x)$. At higher concentrations $C>C_{2}$ we enter a new region ( $E$ on Fig. 3).

In region (E) all correlation lengths are smaller than the slit thickness $D$ and the local correlations are thus identical to those of a bulk solution. We may define the border $C_{2}(x)$ by either one of the following conditions

$$
\begin{aligned}
& \xi_{2}\left(C_{2}\right)=D \\
& \xi_{3}\left(C_{2}\right)=D .
\end{aligned}
$$

Let us choose for instance eq. (3.11) as our starting point. Using eqs. (3.5) and (3.2) we can transform it into

$$
\frac{C_{2}}{C_{1}}=\left(\frac{R_{\mathrm{F} 2}}{D}\right)^{2 / 3} \cong N^{1 / 2}\left(\frac{a}{D}\right)^{5 / 6}
$$

where we have used eq. (2.5) for $\boldsymbol{R}_{\mathrm{F} 2}$. Inserting now the explicit form (3.2) of $C_{1}$, we reach

$$
C_{2} a^{3} \cong\left(\frac{a}{D}\right)^{4 / 3} \text {. }
$$

Inserting this value into the formula for $\xi_{3}$ (eq. (3.4)) we check immediately that (3.12) is equivalent to (3.11). We can also see from the definitions of $x$ and $y$ that (3.12) corresponds simply to $x=y$, i.e. to the first bisectrix in figure 3 .

Having thus specified the border of region E, let us now describe its main properties.

a) The local correlations are isotropic and identical to those of a usual 3 dimensional solution. The correlation length is $\xi_{3}(C)$ (eq. (3.4)).

b) Each chain occupies a region with the shape of a pancake of thickness $D$ and radius $R$. Of course different chains overlap. The radius $R$ may be derived from the blob concept : one chain is made of a sequence of $\mathrm{N} / \mathrm{g}$ blobs, independent of each other, and each of size $\xi_{3}$. Thus

$$
R^{2} \cong \frac{N}{g} \xi_{3}^{2} \cong N a^{2}\left(C a^{3}\right)^{-1 / 4}
$$

where $g=C \xi_{3}^{3}$ is the number of monomers in one blob. Eq. (3.15) is identical in form with what is expected in three dimensional bulk solutions [4]; the numerical coefficient may however be different.

It is possible to check that eq. (3.15) matches smoothly with eq. (3.6) on the borderline $(x=y)$ between regions $\mathrm{D}$ and $\mathrm{E}$.

c) The osmotic pressure $\pi(C)$ is dominated by local correlations and thus retains the 3 dimensional value (eq. (3.4b)). Also, for the equilibrium between a bulk solution and a slit, it may be checked from the scaling form of the chemical potentials that the two concentrations $C_{\mathrm{b}}$ and $C$ are nearly equal. 
d) It is also of interest to discuss the density profile across the slit when $\xi_{3}<D$. We know that in 3dimensional solutions the effects of local perturbations are screened out at distances $r>\xi_{3}$ [4]. Thus the density must vanish on both walls, but must return to the average value $C$ at a healing distance of order $\xi_{3}$ from each wall. In most of the slit the density profile is flat. This differs from the classical results $\left({ }^{4}\right)$ for ideal chains squeezed in a slit, where the healing length is of order $l$. Some readers might be surprised by this difference, since our interacting chains are gaussian on large scales.- But in fact the situation is familiar with concentrated three dimensional solutions : here the osmotic compressibility is low, and as a result the healing length is small [4]. But the large repulsion energies do not prevent the chains from being ideal as first noticed by Flory.

4. Overlapping chains in a capillary. - 4.1 SPECIFIC FEATURES OF THE ONE DIMENSIONAL PROBLEM. - Polymer solutions in three or two dimensions obey a fundamental theorem first realised by Flory [10] : namely, at high concentrations, the chains become ideal. For the slit problem considered in the last section, this implied the existence of a regime which we called semi-dilute with two dimensional correlations. In the diagram of figure 2 this was associated with region (D).

In one dimension the Flory theorem breaks down : self avoiding walks on a one dimensional lattice are always fully extended. This, as we shall see, implies that there is no analog to region (D) : a semi-dilute regime with one dimensional correlations does not exist.

The situation can again be described in a diagram with coordinates $x=R_{\mathrm{F}} / D$ and $y=R_{\mathrm{F}} / \xi_{3}$ (Fig. 3). Here the two boundary lines SM and SL collapse

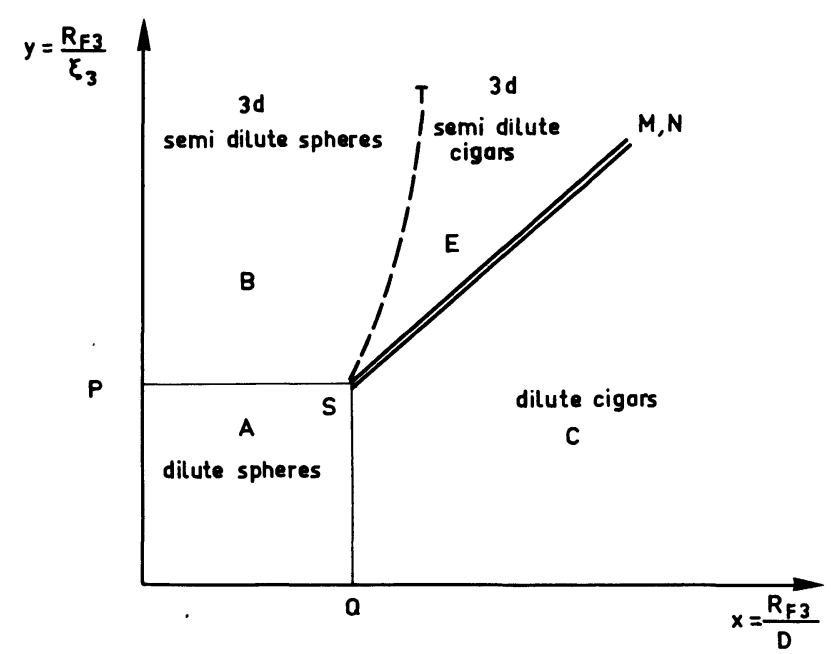

FIG. 3. - Regimes for macromolecular solutions in a capillary. Note the difference with the case of a slit (Fig. 2) : region (D) has disappeared.

(4) See for instance Dolan, A. K., Edwards, S. F., Proc. $R$. Soc. A 337 (1974) 509. into a single line. The cross-over through this double line will turn out to be less smooth than in the preceding cases.

4.2 THE OVERLAP CONCENTRATION $C_{1}(x)$. - On figure 3 the onset of overlap between chains is associated with the line PSM.

a) When the capillary is large $\left(D>R_{\mathrm{F} 3}\right.$ or $\left.x<1\right)$ we return to the familiar case of bulk solutions and we can write

$$
C_{1}(x)=C^{*} \cong \frac{N}{R_{\mathrm{F} 3}^{3}} \sim a^{-3} N^{-4 / 5}(x<1) .
$$

b) For smaller capillaries $(x>1)$ the volume occupied by one coil is of order $D^{2} R_{\mathrm{F} 1}$ where $R_{\mathrm{F} 1}$ is the length of one isolated cigar given in eq. (2.11). The overlap threshold is thus

$$
\begin{aligned}
C_{1}(x) \cong \frac{N}{D^{2} R_{\mathrm{F} 1}} & \cong a^{-3}\left(\frac{a}{D}\right)^{4 / 3} \\
& \cong C^{*} x^{4 / 3} .
\end{aligned}
$$

We meet now the fundamental property : on the line SM defined by eq. (4.2) the three dimensional correlation length $\xi_{3}$ becomes equal to $D$

$$
\xi_{3}\left(C_{1}\right) \cong R_{\mathrm{F} 3}\left(\frac{C^{*}}{C_{1}}\right)^{3 / 4} \cong R_{\mathrm{F} 3} x \cong D .
$$

Above the line $\operatorname{SM}\left(C>C_{1}\right) \xi_{3}(C)$ is smaller than $D$ : the polymer solution must then display local correlations which are three dimensional in character. As announced, we do not find any analog to region (D) of figure 3. We shall now discuss in more detail the properties of these overlapping chain systems (i.e. region $(\mathrm{E})$ in the diagram).

4.3 Semi-DILUTE cigars. - The main physical features expected in region (E) are the following :

a) on a spatial scale $\xi_{3}(C)$ the correlations are not different from what we find in bulk solutions at the same concentration. The osmotic pressure is still given by eq. $(3.4 b)$; and

b) however the overall shape of one coil is not spherical, but elongated, with a certain length $R(C)$ along the capillary axis. The value of $R(C)$ can be derived by the following argument :

Let us consider the density-density correlation function $\tilde{p}(12)$ for one labelled chain in the solution. Physically we may think of $\tilde{p}$ as the density of labelled monomers at point 2 when one labelled monomer is fixed at point 1 .

- For $r_{12}<\xi_{3}(C), \tilde{p}(r)$ has an excluded volume behaviour [4]

$$
a^{3} \tilde{p}\left(r_{12}\right) \sim\left(\frac{a}{r_{12}}\right)^{4 / 3} .
$$

Eq. (4.4) was first obtained long ago by Edwards [13]. 
- For $\xi_{3}(C)<r<D, \tilde{p}\left(r_{12}\right)$ may be derived from the blob concept and decreases like $1 / r$, as in an ideal chain

$$
a^{3} \tilde{p}\left(r_{12}\right) \sim \frac{g}{\xi_{3}^{2} r_{12}}
$$

where $g=C \xi_{3}^{3}$ is the number of monomers in one blob.

- For $r_{12}>D$ the function $\tilde{p}$ must become essentially constant $\left({ }^{5}\right)$ and equal to the average density of labelled monomers for one cigar

$$
\tilde{p}\left(r_{12}\right) \cong \frac{N}{R D^{2}}\left(D<r_{12}<R\right) .
$$

Writing that (4.5) and (4.6) must agree when $r_{12}=D$, we are led to

$$
\frac{C \xi_{3}}{D}=\frac{N}{R D^{2}}
$$

or

$$
R=\frac{N}{C \xi_{3} D} .
$$

Eq. (4.7) gives a radius $R$ linear in $N$ as expected for a one dimensional confinment. However, because of the factor $D$ in the denominator, $R$ is much smaller than for a fully extended chain.

Or course, if at fixed concentration we increase $D$, $R$ decreases, and at some moment, $R$ becomes equal to $D$ : we then cross-over to a conventional bulk solution. This corresponds to the line ST in the diagram of figure 3. In order to locate the line ST we may write that $D$ is equal to the radius $R_{3}(C)$ derived in ref. [4] for three dimensional solutions. $R_{3}(C)$ is in fact given by our eq. (3.15) and thus we have on the line ST

$$
D=R_{3}(C)=R_{\mathrm{F} 3}\left(\frac{C^{*}}{C}\right)^{1 / 8} .
$$

This may also be written

$$
x=\frac{R_{\mathrm{F}}}{D} \cong\left(\frac{C_{7}}{C^{*}}\right)^{1 / 8}
$$

or

$$
y=x^{6} .
$$

Eq. (4.9) may in fact be used as a starting point for another derivation of the cigar length in region $E$. Since $R / R_{\mathrm{F} 3}$ depends only on the reduced variables $x$ and $y$ we expect to have

$$
R \cong R_{\mathrm{F} 3} x^{u} y^{v} .
$$

- Ori the line $\operatorname{SM}(x=y)$ we must have

$$
R=R_{\mathrm{F} 1}=R_{\mathrm{F} 3} x^{2 / 3} .
$$

Thus $u+v=2 / 3$.

$\left({ }^{5}\right)$ This may be stated more precisely by saying that $\tilde{p}(R) / \tilde{p}(D)$ is a numerical constant of order unity.
- On the line $\operatorname{ST}\left(y=x^{6}\right)$ we must have $R=R_{3}(C)$, giving

$$
\frac{u}{6}+v=-\frac{1}{6} \text {. }
$$

This gives $u=1$ and $v=-1 / 3$, or equivalently

$$
R=R_{\mathrm{F} 3} x y^{-1 / 3} \text {. }
$$

It is easily seen from the definitions of $x$ and $y$ that this agrees with (4.7).

Finally, we may mention a pictorial derivation of (4.7) which is rather illuminating. This is based on the notion of superblobs of size D. Each superblob contains a number $\left(D / \xi_{3}\right)^{2}$ of consecutive blobs along one chain. The factor $\left(D / \xi_{3}\right)^{2}$ expressing the fact that the blob sequence is ideal for spatial scales $r$ such that $\xi_{3}<r<D$. The number of monomers in one superblob is thus

$$
g_{\mathrm{s}} \cong g\left(\frac{D}{\xi_{3}}\right)^{2}
$$

and the number of superblobs in one chain is $N / g_{\mathrm{s}}$ Each superblob is disposed consecutively to the next one. Thus the overall length is

$$
R \cong \frac{N}{g_{\mathrm{s}}} D=\frac{N \xi_{3}^{2}}{g D}=\frac{N}{C \xi_{3} D} .
$$

Consider now the process where we decrease the size of the capillary at fixed concentration (fixed $y>1$ ) and enter the region $\mathrm{E}$ of the diagram : the chain radius starts from the value $R_{3}(C)$, and then increases linearly with $x$ according to eq. (4.10). Finally, when we cross the line $\operatorname{SM}(x=y)$ the radius reaches the value $R_{\mathrm{F} 1}$. It is of importance for thermodynamic purposes, to calculate the entropy change $\Delta S$ associated with this stretching process. Quite generally for one chain of independent blobs we would expect

$$
-\Delta S / \text { chain } \cong \frac{R^{2}(C)}{R_{3}^{2}(C)} .
$$

In the present one dimensional problem we would have to supplement this by an interaction term (since superblobs cannot be treated as independent, and this is precisely the source of the stretching). The radius $R$ results from an optimisation of the sum $((4.13)+$ interaction term). As usual however the two terms will be comparable after optimisation, and we can retain (4.13) as the whole work (divided by $k_{\mathrm{B}} T$ ) required to bring one chain into region $\mathrm{E}$ from the bulk solution at concentration $C$. In dimensionless variables this is

$$
-\Delta S / \text { chain } \cong x^{2} y^{-1 / 3} .
$$

Note that this crosses over smoothly to the value $-\Delta S=x^{5 / 3}$, which was given in eq. (2.8) for separate cigars, when $x=y$. 
It can be checked that $T \Delta S$ is always a small correction to the free energy per chain : thus the equation for the osmotic pressure is not modified significantly by stretching. Let us now write down chemical potentials and equilibrium conditions between phase $E$ and a bulk solution.

The chemical potential $\mu_{\mathrm{E}}$, in phase $\mathrm{E}$, per monomer, is of the form :

$$
\begin{aligned}
& \mu_{\mathrm{E}}=\mu_{0}+\mu_{1}+\mu_{2}+\mu_{3} \\
& \mu_{1} \cong k_{\mathrm{B}} T N^{-1} x^{5 / 3} \\
& \mu_{2} \cong k_{\mathrm{B}} T N^{-1} x^{2} y^{-1 / 3} \\
& \mu_{3} \cong \frac{\pi}{C} \cong k_{\mathrm{B}} T N^{-1} x^{5 / 3}
\end{aligned}
$$

where $\mu_{1}$ is the squeezing term for a single chain, $\mu_{2}$ is the lateral stretching term discussed above, and $\mu_{3}$ is the effect of repulsions in bulk 3-dimensional solutions. The ratio

$$
\frac{\mu_{2}}{\mu_{1}} \cong\left(\frac{x}{y}\right)^{1 / 3}
$$

is small in region (E), and $\mu_{2}$ can be omitted. We write the equilibrium condition between the confined solution and a bulk solution of concentration $C_{\mathrm{B}}$. The bulk chemical potential is from eq. (3.8)

$$
\mu_{\mathrm{B}}=\mu_{0}+\mu_{3}\left(C_{\mathrm{B}}\right) .
$$

Writing $\mu_{\mathrm{E}}=\mu_{\mathrm{B}}$ we find that $C$ is only slightly smaller than $C_{\mathrm{B}}$, the relation between the two being

$$
\left(C_{\mathrm{B}} a^{3}\right)^{1.25}-\left(C a^{3}\right)^{1.25} \cong N^{-1} x^{5 / 3} .
$$

Returning now to the problem of chain conformation in region $\mathrm{E}$, we shall now consider briefly the number $v$ of distinct chains cutting one chosen cross section of the capillary. This is roughly equal to the chain concentration $C / N$ multiplied by the chain length $R$ and by the cross-section area $D^{2}$

$$
\begin{aligned}
v & =\frac{C}{N} D^{2} R \\
& =\frac{D}{\xi_{3}} .
\end{aligned}
$$

At the overlap concentration (where we know from (4.4) that $\xi_{3} \cong D$ ) we have $v \cong 1$ as expected. On the other hand, for molten polymers $\left(\xi_{3}=a\right) v$ is of order $D / a$ : note that this value is much smaller than what we would have for a bundle of completely elongated chains $\left(v \sim D^{2} / a^{2}\right)$. These considerations on $v$ might be of use for discussions of the rheological behaviour of solutions in fine pores.

4.4 ANOMALIES NEAR THE OVERLAP CONCENTRATION. - As pointed out earlier, the limiting line SM is really the result of the collapse of two lines SM and $\mathrm{SN}$ : thus the cross-over properties here are somewhat unusual. The cigar length $R$ crosses over smoothly between eqs. (2.11) and (4.7). However, the osmotic pressure $\pi$ does not. A qualitative plot of $\pi(C)$ near $C=C_{1}(x)$ is shown on figure 4. Below the threshold we expect to have something like the Tonk's equation [14] for hard rods in one dimension

$$
\frac{\pi(C)}{k_{\mathrm{B}} T} \sim \frac{C}{N} \frac{C_{1}}{C_{1}-C} \quad\left(C<C_{1}\right) .
$$

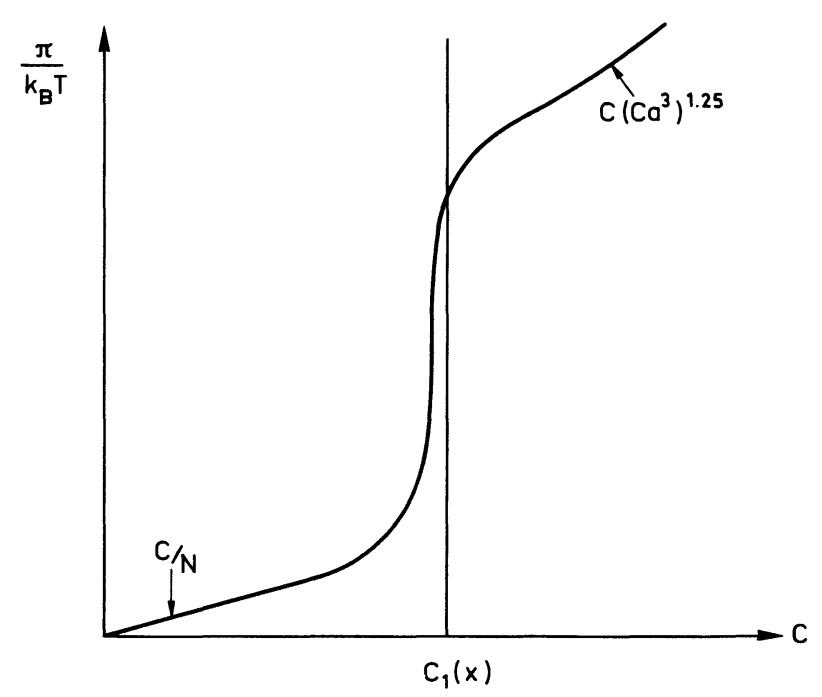

FIG. 4. - Reduced osmotic pressure $\pi / k_{\mathrm{B}} T$ versus concentration $C$ near the overlap threshold $C_{1}$, for a system of chains trapped in a capillary. Note that the limiting forms far above and below $C_{1}$ do not join in smoothly.

Above $C=C_{1}$ we expect to find the three dimensional law $(3.4 b)$. It must be realized that

$$
\frac{C_{1}}{N} \ll C_{1}\left(C_{1} a^{3}\right)^{1.25} .
$$

(Equality occurs only when $C_{1}=C^{*}$, i.e. at point S.) This is completely different from what we know in two or three dimensions : in one dimension, separate cigars find it very difficult to interpenetrate, and the pressure must rise far above $C_{1} / N$ before crossing over to the semi dilute value.

We may add at this point one comment for readers which are familiar with the magnetic phase transition analog [7] : the $n$ vector model, when extrapolated to the non physical value $n=0$, has a finite transition temperature in one dimension [15]. However, the critical exponents are unusual and in particular the magnetisation exponent $\beta$ vanishes. The unusual features of $\pi(C)$ are related to this anomaly.

5. Concluding remarks. - We have reached a certain qualitative understanding of the static conformation of flexible coils confined into small pores. This description is powerful, because it includes the excluded volume effects with the same level of accuracy which is reached in the Flory theory of a single coil. On the other hand, the results are weak because they 
lack precise numerical coefficients. But the scaling laws involving the dimensionless parameters $x$ and $y$ are interesting and will hopefully be checked by experiments.

The present work can be extended in various directions :

a) The dynamics of confined polymer solutions is of great interest, and can be attacked by scaling methods $\left({ }^{6}\right)$ in analogy with recent work on bulk solutions [12].

b) The problems of adsorption of a polymer on a surface are complex but interesting. A scaling theory

$\left(^{6}\right)$ Brochard, F., Private Communication. of reversible (weak) adsorption of chains on one flat surface has recently been proposed [16]. The main limitation here is apparently the difficulty of reaching thermal equilibrium in the adsorption process.

c) A somewhat different class of questions is connected with systems where the boundaries are not smooth e.g. chains trapped inside a gel. Here, however we shall face problems of gel statistics which are one order of magnitude more difficult from the theoretical standpoint.

Acknowledgments. - We thank G. Jannink and P. Pincus for various discussions on confined chain statistics, and the referee for correcting a serious mistake in eq. (4.15).

\section{References}

[1] For a general introduction see.

Colton, C. K., SAtTerfield, C. M., LaI, C. J., Diffusion and partitioning of macromolecules within finely porous glass AIChEJ 21 (1975) 289.

[2] Satterfield, C., Katzer, J. R., Adv. Chem. Series 102 (1971) 193.

Satterfield, C., Cheng, C. S., AIChE 18 (1972) 724.

MoOre, R., Katzer, J. R. AIChE 18 (1972) 216.

[3] LAI, C. J., Sc. D. Thesis (MIT 1973).

[4] Daoud, M., Cotton, J. P., Farnoux, B., Jannink, G., Sarma, G., Benoit, H., Duplessix, R., Picot, C., DE Gennes, P. G. Macromolecules 8 (1975) 804.

[5] Casassa, E. F., J. Polym. Sci. B 5 (1967) 773.

Casassa, E. F., Tagami, Y., Macromolecules 2 (1969) 14.

[6] De Gennes, P. G., Phys. Lett. 38A (1972) 339.

[7] Des Cloizeaux, J., J. Physique 36 (1975) 281.

See also the appendix to ref. [4].

[8] Fisher, M. E., BARber, M. N., Phys. Rev. Lett. 28 (1972) 1516.

[9] Binder, K., Hohenberg, P. C., Phys. Rev. B 6 (1972) 3461.

[10] Flory, P., Principles of polymer chemistry (Cornell Univ. Press Ithaca, N.Y.) 1953.

[11] Des Cloizeaux, J., J. Physique to be published.

[12] De Givis, P. G., Macromolecules 9 (1976) 587, 594

[13] Edwards, S. F., Proc. Phys. Soc. 85 (1965) 613.

[14] Lieb, E. H., Mattis, D. C., Mathematical Physics in one dimension (Academic Press) 1966.

[15] Balian, R., Toulouse, G., Ann. Phys. 83 (1974) 28.

[16] De Gennes, P. G., Euchem. Conference on Interfaces (Collioure) 1976. 